\title{
The Lady's not for turning: Rotation of the Thatcher illusion
}

\author{
Michael B Lewis
}

School of Psychology, Cardiff University, POBox 901, Cardiff CF103YG, UK; e-mail: LewisMB@Cardiff.ac.uk Received 22 December 2000, in revised form 1 March 2001

\begin{abstract}
Inversion is especially detrimental to the processing of faces. This is clearly demonstrated by the Thatcher illusion. It has been suggested that this detriment is due to a loss of holistic or configural processing for inverted faces (Bartlett and Searcy, 1993 Cognitive Psychology 25 281 -316). Stürzel and Spillmann (2000 Perception 29 937-942) suggest that this loss of configural processing occurs suddenly as a face is rotated slowly from upright to inverted. This hypothesis is tested in a study of the reaction times taken to indicate that a face has been Thatcherised at various angles of orientation. The results suggest that there is a gradual loss of configural information rather than a rapid switch from one type of processing to another.
\end{abstract}

\section{Introduction}

Inverting the eyes and mouth of a face makes it look grotesque. Inverting this whole image removes this grotesque appearance. This effect, known as the Thatcher illusion (Thompson 1980), has been of considerable use in understanding the consequences of inversion on face processing.

The Thatcher illusion has been explained in terms of the two different encoding processes thought to underlie face recognition. Tanaka and Farah (1993) demonstrated how faces, which may be encoded according to component features (eyes, nose, etc), are more usually encoded according to holistic or configural features (ie the relationship between component features). This distinction between configural and componential encoding, however, is far from clear and well defined (eg a componential feature like a large chin can equally be seen as a configural feature as the distance between mouth and bottom of the face). The configural features can be operationally defined as those features of a face that are disrupted by inversion. Turning a face upside down, therefore, makes recognition only possible from the component features.

Bartlett and Searcy (1993) described how component and configural features could explain the Thatcher illusion: Thatcherising a face changes only the configuration of the features. These make the face look odd but only when encoded by configural features. The inverted Thatcherised face does not look odd because the configural information (which has been changed) is not available to the viewer.

It has been proposed, therefore, that the Thatcher illusion can be used to test for configural processing. Lewis and Johnston (1997) used the Thatcher illusion to test whether inversion of the brightness pattern of an image (ie a photographic negative) disrupts configural encoding, and found that it does-but not to the same degree as inversion. This experiment, therefore, provides evidence that the disruption of configural encoding is not necessarily all or none. It is possible, in fact, to transform a face such that configural encoding is disrupted only to a degree.

Stürzel and Spillmann (2000) investigated whether, during rotation, there is a point at which configural encoding is no longer useful. They also investigated at what angle of rotation such a switch in configural encoding might take place. The conclusions drawn from this experiment (a shape switch at about $95^{\circ}$ ) are contrary to the idea of levels of disruption of configural encoding (implicit from the finding of Lewis and Johnston 1997) and so their experiment needs to be considered carefully. 
Stürzel and Spillmann (2000) placed three Thatcherised faces individually on a rotating disc and asked subjects to say when the face appeared to change from normal to grotesque or from grotesque back to normal. They found that the change occurred somewhere between a rotation of $97^{\circ}$ (normal to grotesque) and a rotation of $118^{\circ}$ (grotesque to normal). As these add up to more than $180^{\circ}$ there was an amount of overlap. This overlap may be a result of some sort of hysteresis as is known to occur with threshold-measurement techniques, which use the method of ascending and descending limits, but one cannot discount the fact that the overlap may be a result of the methodology employed. The faces were rotated at a speed of $30^{\circ}$ per second and so the time taken to stop rotation (one would expect this to take at least 0.5 to $1 \mathrm{~s}$ ) could lead to an overestimation of between $15^{\circ}$ and $30^{\circ}$ in each direction.

These results are used by Stürzel and Spillmann (2000) to highlight that the face processing 'switches' from configural to a more component form of encoding at a particular degree of rotation. This conclusion, however, does not necessarily follow from their data as they asked their subjects to report when a switch occurred and then used these reports to conclude that a switch did indeed occur. In this way, they forced the subjects to define a point where there was a change even if, in fact, the actual change they observed was gradual.

The conclusion of a switch between configural and component encoding was further supported, it was claimed, by the research conducted by Sjoberg and Windes (1992). In their study, they also looked at the Thatcher illusion at various angles of rotation but this time the subjects' task was to indicate, as quickly as possible, whether the face was Thatcherised or not. The angles of rotation were in steps of $60^{\circ}$ and the stimuli they used were Mac-a-Mug faces.

Sjoberg and Windes' (1992) results showed that the time to make a Thatcherisation decision increased as the angle of rotation from upright increased. They also found that there was no significant interaction between orientation and type (Thatcherised versus normal). Sjoberg and Windes concluded, therefore, that the strategies did not differ for the two types of faces and, further, that "mental rotation is essentially the same for faces as non-face stimuli" (page 1178). One problem with this conclusion, however, is that the experiment used Mac-a-Mug faces and not real faces. The conclusion should be that "mental rotation is essentially the same for [Mac-a-Mug] images as non-face stimuli". This statement does not have the same generalisability as the original. The increase in reaction times between $60^{\circ}$ and $120^{\circ}$ is larger than any other change but no statistics were conducted to test whether, as Stürzel and Spillmann (2000) conclude, this really is a stepped function. Indeed, Sjoberg and Windes conclude that there is not a discontinuity in the reaction-time functions.

The conclusions from Sjoberg and Windes (1992) and Stürzel and Spillmann (2000) are clearly at odds in some respects. The former suggest that in order to see that a face is Thatcherised it must be rotated and then inspected. The latter suggest that there is a switch from configural to component encoding as angle increases and, as Thatcherisation is difficult to detect from purely component features, after this switch the Thatcherised face looks normal. The former suggest that there is a gradual increase in reaction times to spot a Thatcherisation as angle of rotation increases whereas the latter believe that the same data show a discontinuity or switching somewhere between $60^{\circ}$ and $120^{\circ}$.

This issue of a discontinuity of reaction times to spot a rotated Thatcherised face was investigated in the experiment presented here. The experiment was similar to that of Sjoberg and Windes (1992) in many respects, however, there are a number of differences. First, the images used were real faces as opposed to Mac-a-Mug faces. This means that the results inform us about face processing rather than the processing of more artificial images. Second, all the images were of famous individuals. The original Thatcher 
illusion was shown by using a famous individual's face and so it was appropriate to use famous faces in this experiment. Third, rotation was performed in steps of $10^{\circ}$. These steps are six times smaller than the steps used by Sjoberg and Windes and so provide a much finer level of detail. If indeed there is a discontinuity in reaction times then this should be apparent from a sharp increase in reaction times over three or four steps.

\section{Method}

\subsection{Subjects}

Forty undergraduates from Cardiff University received course credit for their participation in this experiment.

\subsection{Stimuli}

Five front-view images of famous actors (Leonardo DiCaprio, Brad Pitt, Harrison Ford, Keanu Reeves, and Bruce Willis) were digitally scanned and represented in 256 colours. Each of the five stimuli faces were Thatcherised with image-processing software. This procedure involved selecting rectangles around each eye and the mouth. Each of these rectangles was inverted and any sharp colour changes were smoothed with a smudge tool. The resulting ten stimuli were then rotated through $350^{\circ}$ in steps of $10^{\circ}$ to generate the 360 stimuli that were presented to the subjects.

\subsection{Procedure}

Subjects were introduced to the Thatcher illusion with examples not used in the experiment. They then sat in front of a computer monitor and were presented with the 360 stimuli in individual trials. Each trial began with the presentation of a face or a Thatcherised face and ended when the subject made a key press. The subject was required to indicate whether the faces were Thatcherised or normal by pressing one of two keys. The next trial began $1 \mathrm{~s}$ after the subject made a response to the last trial. Trials were presented in a randomised order individually generated for each subject.

\section{Results}

The reaction times (once any errors had been removed) were split according to the degree of rotation from the normal orientation and whether the faces were normal or Thatcherised. The mean reaction times are shown in figure 1 . The plots for the Thatcherised and the normal faces each show a general increase as the image is rotated but this increase is not linear. It would appear that the plots are relatively flat

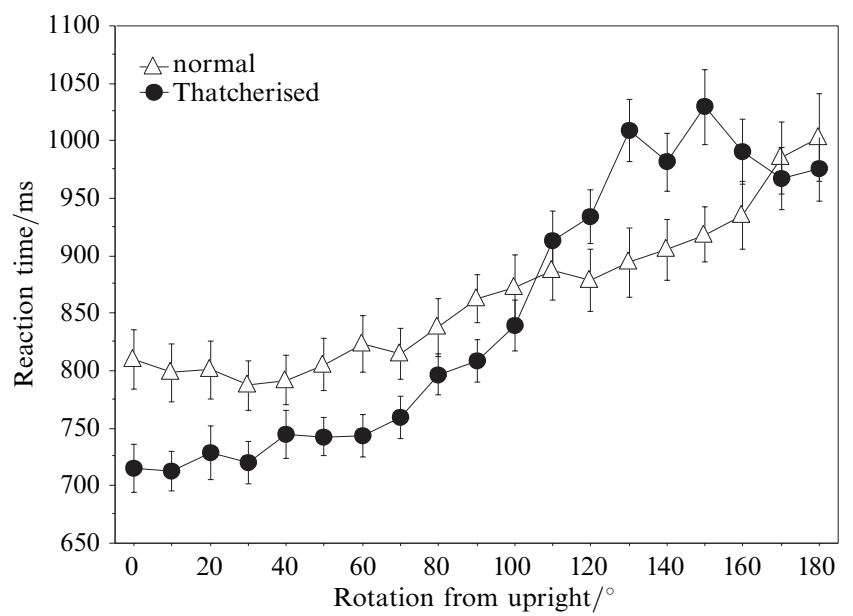

Figure 1. Reaction times to indicate that an image is either a Thatcherised face or a normal face. Error bars show standard error. 
until between $60^{\circ}$ and $90^{\circ}$. The plots then rise more steeply (with Thatcherised faces having a steeper gradient than normal faces). The reaction times for Thatcherised faces then level off after between $130^{\circ}$ and $160^{\circ}$ and the plots come together for inverted items. The reaction times for normal faces continue to rise all the way through to $180^{\circ}$, at which point the times are comparable to those for inverted Thatcherised faces.

An ANOVA was conducted over the reaction times and revealed a significant effect of degree of rotation (by items $-F_{18,72}=112.192, p<0.001$; by subjects$F_{18,702}=15.940, p<0.001$ ) and a significant interaction (by items $-F_{18,72}=8.017$, $p<0.001$; by subjects $-F_{18,702}=5.741, p<0.001$ ). The effect of type of face (normal or Thatcherised) was not significant (by items $-F_{1,4}<1$; by subjects $-F_{1,39}=2.467$, $p>0.05$ ). In order to investigate the apparent nonlinear nature of the reaction times, two polynomial regressions (to the third power) were conducted (one for normal faces and one for Thatcherised faces). The regression for normal faces gave a significant linear component $\left(\beta=0.955, t_{15}=8.112, p<0.001\right)$ and a significant quadratic component $\left(\beta=0.279, t_{15}=5.995, p<0.001\right)$ but a nonsignificant cubic component $\left(\beta=-0.0108, t_{15}=0.108, p>0.05\right)$. The regression for Thatcherised faces gave a significant linear component $\left(\beta=1.625, t_{15}=11.334, p<0.001\right)$, a nonsignificant quadratic component $\left(\beta=0.040, t_{15}=0.709, \quad p>0.05\right)$, and a significant cubic component $\left(\beta=-0.760, t_{15}=5.302, p<0.001\right)$. Both regressions, therefore, show significant deviation from a linear increase in reaction times.

\section{Discussion}

The results from the experiment show some common features with the results found by Sjoberg and Windes (1992) but there are also some important differences. A similarity, for example, was that the reaction time to make a Thatcherisation decision increased as the image was rotated from upright to inverted. This was true regardless of whether the face was Thatcherised or not. One important difference was that this experiment found an interaction between type of face and angle of rotation such that, when upright, decisions to Thatcherised faces were faster than decisions to normal faces but when faces were inverted there was almost no difference.

While the data do show a steeper increase between $60^{\circ}$ and $120^{\circ}$ than between $0^{\circ}$ and $60^{\circ}$ [as did Sjoberg and Windes' (1992) data] there is no evidence of a discontinuity between $60^{\circ}$ and $120^{\circ}$. The increases in reaction times appear relatively constant over this range. The polynomial analysis demonstrates that the effect of rotation on reaction times cannot be interpreted as being linear. Such a nonlinearity would be taken by Sjoberg and Windes to imply that the rotation of faces is special compared with other objects [Shepard and Metzler (1971) found that object recognition time was linearly related to degree of rotation]. Faces are not simply being rotated in order to determine whether or not they have been Thatcherised but instead there are different types of processing taking place for upright than for inverted images. This is consistent with the accumulation of evidence that inversion disrupts fast configural encoding of faces, leaving processing to take place by slower component encoding (eg Tanaka and Farah 1993).

The lack of a discontinuity in reaction times is in contrast to Stürzel and Spillmann's (2000) interpretation of how configural processing is affected by rotation. Stürzel and Spillmann used their experiment, which asked subjects to report switching, to conclude that there is a degree of rotation beyond which configural processing cannot be employed. A failure to find this discontinuity is at odds with Stürzel and Spillmann's interpretation. The change from configural processing of upright faces to purely component processing of inverted faces appears to be gradual as an image is rotated, leading to a gradual increase in reaction times required to spot a Thatcherised face. The results of Stürzel and Spillmann's experiment therefore need to be explained 
within the proposed framework of a gradually decreasing contribution of configural encoding through rotation. One clue to an explanation comes from the fact that there is a crossover in the two reaction-time curves at a rotation of $105^{\circ}$, which is similar to where Stürzel and Spillmann found their switches to occur (between $94^{\circ}$ and $100^{\circ}$ ). Exactly how this crossing of reaction-time curves relates to 'grotesque/pleasant' decisions is difficult to explain but one might be able to envisage the situation where subjects make such a decision based on whether they think it would be quicker to say that a face at that orientation was Thatcherised or not. This relationship between the two sets of data is speculative but may be worthy of further consideration.

Whilst it would appear that the data from the current experiment indicate that configural encoding is gradually degraded through rotation, this is not the only possible conclusion. It is possible that the data of the current experiment represent an averaging over a discrete switching of processing style. This would imply that for some subjects or for some items this switch must take place at about $40^{\circ}$ and for other subjects the switch does not take place until about $160^{\circ}$. Such a large range over which the switch could take place is difficult to explain and inconsistent with the narrow band of change suggested by Stürzel and Spillmann (2000). Further, averaging over a discrete but variable switch would also be apparent in the standard errors of the data as larger standard errors would occur near where the switch is thought to take place. The standard errors shown in figure 1 clearly show that they remain reasonably constant in size across all rotations of Thatcherised and normal faces. The preferred account, therefore, is that there is a gradual degradation in configural encoding as the angle of rotation from upright increases.

A further explanation of the data comes from the fact that the distinction between what is configural and what is componential is not clearly defined. The working hypothesis is that configural features are those that are disrupted by inversion, and it is thought that these reflect holistic features of the face or relational aspects of features. It is possible, however, that there is a continuum of features ranging from very configural to very componential. As a face is rotated, then the most configural features may be disrupted first. Further rotation would gradually remove the less configural features until only the purely componential features remain for the inverted face. Such an explanation would combine a step-tuned function for each configural feature but show a gradual reduction in configural encoding as the face is rotated. A continuum of features from purely holistic configural to local componential is consistent with the idea of face processing being achieved with Gabor filters of various sizes (eg Kalocsai et al 2000). The larger the Gabor filter, the more holistic the feature and, possibly, the more easily the feature may be disrupted by inversion.

One curious and unexpected finding from the current experiment is the reduction in reaction times to say that a face is Thatcherised between rotations of $150^{\circ}$ and $180^{\circ}$. It is possible that this represents spurious variability in the data and so it is probably best not to make too much of it without replication. If this effect is a real feature of performance, however, then it may reflect different strategies being employed for Thatcherised faces that are inverted to those that have been rotated by $150^{\circ}$. What these different strategies might be are not considered here.

If it is true that configural encoding is disrupted gradually by rotation (as is suggested by the current experiment) then this can be tested in other experiments that show the loss of configural encoding for inversion. For example, the difficulty that people have in recognising parts of chimeric faces (see Young et al 1987) should gradually decrease if the face is rotated. Also, the advantage for recognition of a feature within a whole face (see Tanaka and Farah 1993) should also decrease gradually as the faces are rotated. Investigation with these paradigms at various angles of rotation may clarify further the issue of how rotation affects configural encoding. 
Acknowledgements. I wish to thank Andrea Chadwick for carrying out the experiment and Frank Stürzel and an anonymous reviewer for comments on an earlier version.

\section{References}

Bartlett J C, Searcy J, 1993 "Inversion and configuration of faces" Cognitive Psychology 25281 - 316

Kalocsai P, Malsburg C von der, Horn J, 2000 "Face recognition by statistical analysis of feature detectors" Image and Vision Computing $18273-278$

Lewis M B, Johnston R A, 1997 "The Thatcher illusion as a test of configural disruption" Perception $26225-227$

Shepard R, Metzler J, 1971 "Mental rotation of three-dimensional objects" Science 171701 - 703

Sjoberg W, Windes J D, 1992 "Recognition times for rotated normal and Thatcher faces" Perceptual and Motor Skills $751176-1178$

Stürzel F, Spillmann L, 2000 "Thatcher illusion: Dependence on angle of rotation" Perception $29937-942$

Tanaka J W, Farah M J, 1993 "Parts and wholes in face recognition" Quarterly Journal of Experimental Psychology A $\mathbf{4 6} 225$-246

Thompson P, 1980 "Margaret Thatcher-a new illusion" Perception 9 483-484

Young A W, Hellawell D J, Hay D C, 1987 "Configurational information in face perception" Perception $16747-759$ 